\title{
Theoretical approach to modeling the combustion process in turbine engines fuelled by alternative aviation fuels containing various components/biocomponents
}

The aim of this paper is presentation of the possibility of combustion processes modelling so that to better describe the influence of fuels chemistry on fuels combustion. This is important for prediction the behaviour of different alternative fuels in processes in combustion chamber. Currently used mathematical models do not sufficiently take into account the influence of fuels chemical composition on combustion process. The idea of new mathematical model is proposed in this paper. The paper presents the main assumptions of this model and the results of its preliminary verification using MiniJetRig.

Key words: diesel fuel, jet fuel, mathematical model, combustion process

\section{Introduction}

Intensive development of road and air transport leads to respectively increasing air pollution with exhaust gases from internal combustion engines. At global scale, the most important is $\mathrm{CO}_{2}$ emission, considered as global warming effect cause. At local scale, more important is emission of $\mathrm{CO}$, unburned hydrocarbons, and particle matters, as well as $\mathrm{NO}_{\mathrm{x}}$ emission.

Majority of countries have declared implementation the $\mathrm{CO}_{2}$ emission reduction programs. The fundamental way to achieve the goal is to implement biofuels into road and air transport.

Biofuels and biocomponents have been implemented in road transport for about 20 years. The biocomponents commonly used in fuels are: bioethanol as automotive gasolines component, and FAME as diesel fuel component. The technologies of manufacturing hydrocarbon biofuels designed to be used in CI engines and in aviation turbine engines have been developed for about 10 years. Regarding the chemical structure, the hydrocarbon biofuels are more similar to conventional than bioethanol and FAME. However, the hydrocarbon structure influences the combustion process, hence the operation parameters of engine, and exhaust gas composition.

Research regarding new biocomponents/biofuels, both for CI engines, and for aviation turbine engines demands the development of predicting methods, and assessment the effect of fuels/biofuels chemical composition on combustion process.

The characteristics of combustion process in CI engine indicates strong diversification of reaction chains depending on engine operation conditions, and on energy state of combustion chamber [1]. Similar situation takes place in turbine engines.

Research on dependence of combustion process, and as a consequence, engine operation parameters, and exhaust gases composition, are being carried out at different levels, from macroscopic one (engine tests) to molecular one (tests in laboratory reactors). As a result of such research experimental data are obtained, used then to develop the mathematical models quantitatively describing the relationships between chemical structure of fuels and engine operation parameters as well as exhaust gases composition. The basic problem is how describe quantitatively the chemical struc- ture of fuels, what significantly limits the possibilities of combustion processes modelling.

The aim of this paper is to propose new mathematical model of combustion process, previously developed for another application than combustion process.

\section{The analysis of processes in combustion chamber}

The fuel combustion in CI engines and in turbine ones is complex process. It should be noticed at least the one process taking place before combustion chamber, i.e. in fuelling system and injector - fuel thermal degradation, and three processes in combustion chamber, such as: atomisation of fuel stream, its vaporization together with further thermal degradation, and combustion.

The separation of elementary processes is needed to analyse the effect of fuels chemical structure and resulting fuel properties on whole complex fuel combustion process. However, such distinct division into elementary processes doesn't fully correspond to the identified processes in engine. Especially it regards to fuel vaporisation and combustion. It is obvious that atomised, but still liquid fuel, in contact with the atmospheric oxygen diffusing into the liquid phase is subjected to initial oxidation reactions that make up long and branched chains of combustion reaction.

Also the combustion process itself is not uniform in whole combustion chamber. Such diversity, caused mainly by heterogeneous thermal state of combustion chamber is recognized as significant reason to create various products of combustion including $\mathrm{CO}$, unburned hydrocarbons and particulate matters.

Next topic, very important for simulation, is term "chemical structure of fuel". It is known that conventional (petroleum) fuels are the mixture of many (even a thousand) hydrocarbons of different chemical structure. The molecules of individual compounds interact of each other. Such interactions affects fuel viscosity, its vaporisation azeotrope, as well as chemical reactions during combustion process. However the nature of molecular interactions hasn't been sufficiently recognised. In recent years we can notice some interesting information regarding molecular clusters generated in fuels such as aviation turbine ones and diesel fuels, as a result of intermolecular bonds, including van der Waals ones, between the molecules of hydrocarbon 
of specific chemical structure. Authors of the publication [2] describe generation of molecular clusters by polycyclic aromatic hydrocarbons (PAH), and point the clusters as the soot source. The molecular cluster are ordered structure of unique properties, different from volumetric properties of fuel treated as the liquid. This regards the energy state and ability to energy transfer [2]. The molecular clusters existing in fuel undoubtedly influence the chemical reactions in combustion process. This is pointed, among others, by authors of [3, 4].

Above mentioned complexity of chemical structure of fuels, as well as complexity of individual processes taking place in combustion chamber, makes simulation of combustion process fundamentally difficult.

\section{Currently used mathematical models of combustion process in internal combustion engines}

The mathematical models should be useful to predict the influence of fuels chemical structure on combustion process. The mathematical models of combustion processes are generally based on description of relationship between inputs and outputs. Outputs are engines operating parameters, like engine power, thrust, temperature at different points of engine and composition of exhaust gases. All these parameters are measured and output can be determined quantitatively. The problem is with input - fuels chemical structure, which usually is described qualitatively. The chemical reactions are quantitatively describe by thermodynamic relationships and chemical kinetic once. Thermodynamic description operates the thermodynamic parameters (functions) which describe energy state of fuel as a whole. Kinetic equations use such parameters as concentration of chosen reactant - compound which undergoes given reaction. It is very difficult or even impossible to select individual reaction in case the thousand compounds undergo simultaneously similar reactions. Consequently instead of concentration of each compound consisting of fuel the physicochemical properties of fuel are used to quantitatively describe fuel.

The relationships between input - properties of fuel, and output are formulated using different statistical methods. Usually a great number of tested fuels and engine tests results are needed to formulate function describing this relationship.

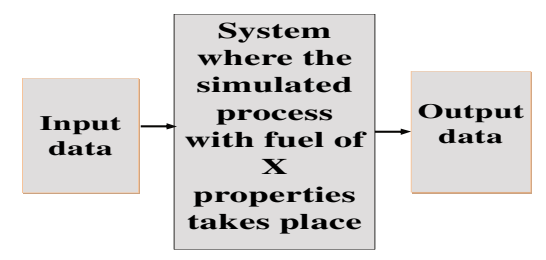

This method of modelling of combustion process uses the measureable parameters characterising fuel - it is advantage of this method. Weakness of this method is that new, unknown components or additives introduced into the fuel can change the mechanism of combustion process what is not included in the model. In this case the important incompatibility of the model with experimental data takes place. Below two of currently use models are briefly presented.

\subsection{The CHEMKIN Model}

The CHEMKIN Model is commercial product. It is used to simulate various chemical reactions, both in chemical process as well as in fuel combustion process in internal combustion engines. To simulate reactions with fuel, the model is supported by fuel data base ANSYS. Using this model, it is possible to calculate thermodynamic effects of fuel combustion, hence the amount of heat, pressure change in combustion chamber as function of crank angle, as well as the flame propagation speed and temperature in combustion chamber. These data are further used in calculation of chemical effects of combustion, i.e. in calculation of kinetics of combustion reactions, and creating products of combustion. The Arrhenius equation has been used for kinetic calculations in CHEMKIN model. In this equation, the temperature is the quantity describing the heat supplied to reaction system (RT). According to $[4,5]$, the average temperature for engine combustion chamber is used in calculations. Such attitude means significant exceptions to the real condition of combustion reaction. It is known [1] that there are different temperatures in different areas of the combustion chamber. It affects the combustion reactions and consequently combustion products, e.g. CO rises mostly by the combustion chamber walls. Accepting the average temperature for whole combustion chamber in CHEMKIN model doesn't accept such effect, so it can lead to significant differences between calculated and experimental $\mathrm{CO}$ concentration in exhaust gases.

Author of the publication [6] used the CHEMKIN model for simulation the combustion process in HCCI engine. Such model needs extensive experimental data base, created basing on model fuels ("model-fuel" or "surrogatefuel"), i.e. the mixtures composed of individual, pure compounds e.g. hydrocarbons. This model covers a lot (up to several hundred) of reactions, making up the chemical reaction chains identified during combustion process. Such reactions have been mostly identified basing on analysis of combustion of individual compounds (mostly hydrocarbons) or their mixtures in reactors. It was found that the reactions identified for individual compound change when another compound is added, e.g. the reaction mechanism determined for pure isooctane has changed after toluene addition. This means major restrictions in using the model which doesn't cover such interaction of fuels components. In many cases, this is the reason for significant discrepancies between the mathematical modelling results and experimental data.

\subsection{The SAE Model}

The SAE model is part of procedure of analysis and assessment of gas emission from aircraft engines [7], and is used to calculate the emission of $\mathrm{CO}_{2}, \mathrm{CO}, \mathrm{NO}_{\mathrm{x}}$ and $\mathrm{C}_{\mathrm{x}} \mathrm{H}_{\mathrm{y}}$ in exhaust gases from aviation turbine engines (jet ones), and in ground applications. This model is based on carbon mass balance. Basis for this model is the following chemical reaction: 
$\mathrm{C}_{\mathrm{n}} \mathrm{H}_{\mathrm{m}} \mathrm{O}_{\mathrm{p}} \mathrm{N}_{\mathrm{q}} \mathrm{S}_{\mathrm{r}}+\mathrm{X}\left[\mathrm{R}\left(\mathrm{O}_{2}\right)+\mathrm{S}\left(\mathrm{N}_{2}\right)+\mathrm{T}\left(\mathrm{CO}_{2}\right)+\mathrm{h}\left(\mathrm{H}_{2} \mathrm{O}\right)\right.$ $\left.+\mathrm{U}\left(\mathrm{CH}_{4}\right)\right] \rightarrow \mathrm{P}_{1}\left(\mathrm{CO}_{2}\right)+\mathrm{P}_{2}\left(\mathrm{~N}_{2}\right)+\mathrm{P}_{3}\left(\mathrm{O}_{2}\right)+\mathrm{P}_{4}\left(\mathrm{H}_{2} \mathrm{O}\right)+$ $\mathrm{P}_{5}(\mathrm{CO})+\mathrm{P}_{6}(\mathrm{CxHy})+\mathrm{P}_{7}\left(\mathrm{NO}_{2}\right)+\mathrm{P}_{8}(\mathrm{NO})+\mathrm{P}_{9}\left(\mathrm{SO}_{2}\right)$

The model doesn't cover processes taking place in the system, i.e. in engine combustion chamber but is based exclusively on relation analysis: input data (fuel + air) output data (composition of gases from combustion chamber). The relationships used in this model contain many experimentally determined parameters, which values can not be appropriate in case the new, unknown fuels component is tested. The another weakness of this model is that the fuel is characterised by average chemical formula.

Both currently used mathematical models of combustion process describes fuel as one very complex reactant. Consequently it is difficult to predict the influence of new components/biocomponents of fuels on combustion process.

\section{The new proposed mathematical model of fuel combustion process}

Taking into account:

- all mentioned above weakness of currently use mathematical models of combustion process

- useful of models for predicting the influence of new components/biocomponents or additives added to the fuel on combustion process

- need analysis of the influence of new components on the mechanism of combustion process,

the new model is proposed. This model was previously worked out and tested for another then combustion processes applications. Experience has shown that this model can be useful in mechanisms of various processes analysis and, what seems to be most important, let focus on the one chosen compound or component in very complex mixture an, describe this chosen compound / component influence on complex process, like fuel combustion in engine. It is the reason that this model was chosen to verify the possibility of its application for description of combustion processes.

The assumptions of proposed, new mathematical model of fuels combustion are based on model, developed previously for reactions initiated by heat and mechanical forces [8]. The new model allows to describe directly the impact of selected fuels components or additives on combustion process. The parameter that provides information about similarities or differences of compared combustion processes - for instance combustion process involving Jet fuel with tested component and process involving conventional Jet fuel, is reactivity coefficient $\alpha_{\mathrm{i}}$. The basing relationship for this model is the following:

$$
\alpha_{\mathrm{i}}=\left\{\left[\mathrm{f}(\mathrm{y})-\mathrm{f}_{0}(\mathrm{y})\right] /\left[\phi(\mathrm{y})-\phi_{0}(\mathrm{y})\right]\right\} \mathrm{d} \phi(\mathrm{y}) / \mathrm{df}(\mathrm{y})
$$

where combustion process is described by two functions of the same variable $y$, linked to the final state of the process.

The general dependence (1) has been transformed to dependence (2). It was possible due to $\mathrm{f}(y)$ was treated as energy supplied from environment to the system and $\phi(y)$ was treated as a part of kinetic equation - reaction rate constant.

$$
\alpha_{\mathrm{i}}=\left(\mathrm{L}-\mathrm{L}_{0}\right) / \mathrm{A} \exp \left[-\mathrm{E}_{\mathrm{a}} /(\mathrm{RT}+\varepsilon)\right]\left[\left(\mathrm{e}_{0}\right) \cos \left(\mathrm{k}_{2} \mathrm{~L}+\mathrm{k}_{3}\right)\right] \mathrm{t}
$$

$$
\mathrm{C}=1 / \mathrm{A} \exp \left[-\mathrm{E}_{\mathrm{a}} /(\mathrm{RT}+\varepsilon)\right]\left[\left(\mathrm{e}_{0}\right) \cos \left(\mathrm{k}_{2} L+\mathrm{k}_{3}\right)\right] \mathrm{t}
$$

where: $\alpha_{i}-$ relative reactivity coefficient of test fuel e.g. in combustion process, $\mathrm{L}$ - energy supplied from environment to the system - e.g. combustion chamber where the test fuel is being burnt, $\mathrm{L}_{0}$ - energy supplied from environment to the system - e.g. combustion chamber where the reference fuel is being burnt, $\mathrm{T}$ - temperature, $\varepsilon$ - energy supplied to reaction zone, other than heat $(\mathrm{RT})$ - in case of the process is catalytic one, $e_{0}-$ stream of the energy emitted by the solid's surface (catalyst), A, $\mathrm{k}_{2}, \mathrm{k}_{3}$ - constant quantities, $\mathrm{t}$ - time.

This relationship describes energy distribution between environment and the system, where observed chemical reaction takes place. Assuming the concept presented in paper [8], equation describing reactivity coefficient can be shown as an relationship of energy streams: energy stream supplied to the system, forcing the process, and energy stream C, supplied to the set of reagent molecules, initiating the chemical reaction.

$$
\begin{gathered}
\alpha_{\mathrm{i}}=\left(\mathrm{E}_{\mathrm{S}}-\mathrm{E}_{\mathrm{S} 0}\right) / \mathrm{A} \exp \left[-\mathrm{E}_{\mathrm{as}} /(\mathrm{RT} / \mathrm{t}+\varepsilon / \mathrm{t})\right]\left[\left(\mathrm{e}_{0}\right) \cos \left(\mathrm{bE}_{\mathrm{S}}+\mathrm{d}\right)\right] \\
\mathrm{C}_{\mathrm{S}}=\mathrm{A} \exp \left[-\mathrm{E}_{\mathrm{as}} /(\mathrm{RT} / \mathrm{t}+\varepsilon / \mathrm{t})\right]\left[\left(\mathrm{e}_{0}\right) \cos \left(\mathrm{bE}_{\mathrm{S}}+\mathrm{d}\right)\right]
\end{gathered}
$$

where: Es, $\mathrm{Es}_{0}$ - streams of energy supplied to reaction system, accordingly the one having tested reagent, and reference system (index 0) $E_{a s}$ - stream of reaction activation energy, $\mathrm{T}-$ average temperature of reaction system. According to this equation, energy stream determines the possibility of chemical reaction initiation. The values of these functions change with the change of initial state of the process.

The reactivity coefficient $\alpha_{i}$ relatively describes the effect of selected reagent on the rate of changes of reaction systems internal energy due to changes in stream of energy supplied to the system.

The aim of research presented in this paper was to see if this model could be used to describe combustion process.

\subsection{Methodology of the preliminary verification of the model}

The preliminary verification of the proposed model of combustion process was conducted using miniature jet engine, MiniJetRig stand.

The engine operates as reactor in which the combustion process can takes place at various conditions of engine operation. The reactivity coefficient can be determined experimentally by measuring the effect of change in initial energy state of the system on final state, being the result of combustion process.

The MiniJetRig enables temperature measurement in front of the turbine, at different points of combustion chamber, and behind the turbine, as well as fuel consumption, mass flow rate and rotation speed of turbine. The stand is equipped in analytical instruments, which measure the concentration of combustion products, i.e. $\mathrm{CO}_{2}, \mathrm{CO}, \mathrm{C}_{\mathrm{x}} \mathrm{H}_{\mathrm{y}}$, $\mathrm{PM}$, and $\mathrm{NO}_{\mathrm{x}}$ in exhaust gases.

The tests were conducted at the following rotational speed: 39000,70000 and $88000 \mathrm{rpm}$ in accordance with the procedure developed in Air Force Institute of Technology. At each rotational speed the temperature $T_{2}$ and $T_{\text {eg }}$ were measured, as well as mass flow rate mf. 


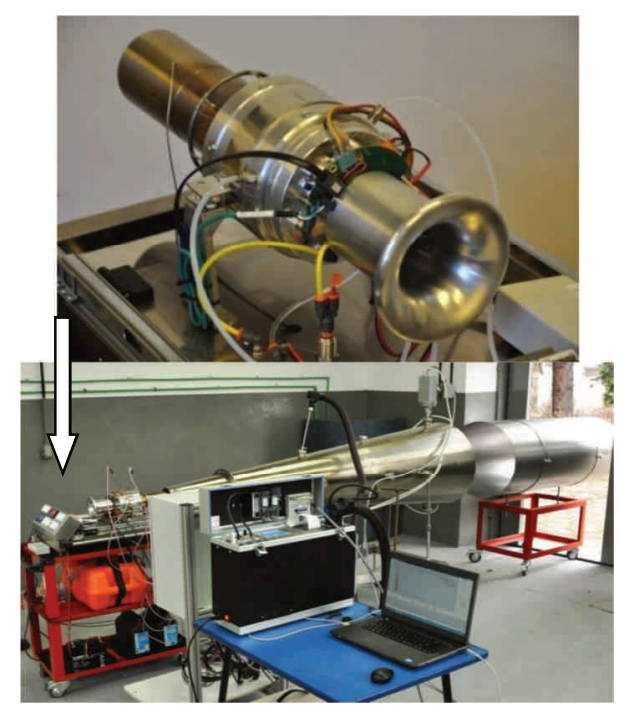

Fig. 1. MiniJetRig testing stand. Source [9]

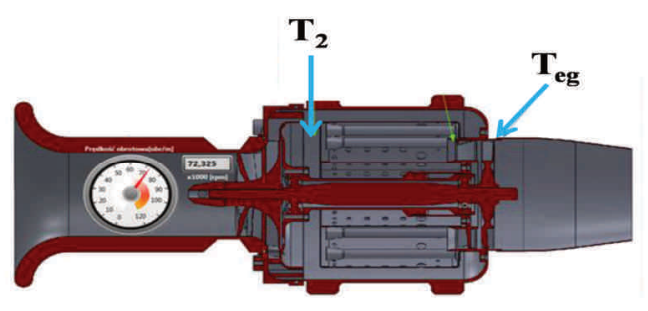

Fig. 2. The scheme of the engine used in verification of the proposed model [9]

The following fuels were tested during preliminary verification of the model:

- conventional mineral Jet A1 as reference fuel

- Blend No. 1 - the mixture of Jet A1 and the blend of synthetic, paraffinic hydrocarbons $\mathrm{C} 15$ and $\mathrm{C} 17$ added together to Jet A1 in concentration of $10 \%(\mathrm{~V} / \mathrm{V})$

- Blend No. 2 - the mixture of Jet A1 and the blend of synthetic, paraffinic hydrocarbons $\mathrm{C} 8$ and $\mathrm{C} 11$ added together to Jet A1 in concentration of $10 \%(\mathrm{~V} / \mathrm{V})$

The experimentally obtained tests results were used to calculate $\alpha_{i}=\alpha_{88 / 39}$ values for tested fuels.

The basic relationship (1), after integration within the limits of the initial and final state of the system gives dependence (6), which was used to calculate $\alpha_{i}=\alpha_{88 / 39}$ values for tested fuels.

$$
\ln \left[\mathrm{F}(\mathrm{y})_{2} / \mathrm{F}(\mathrm{y})_{1}\right]=[1 / \alpha \mathrm{i}] \ln \left[\Phi(\mathrm{y})_{2} / \Phi(\mathrm{y})_{1}\right]
$$

where $\mathrm{y}=\mathrm{b}_{\mathrm{xx}}$

$$
\begin{gathered}
\mathrm{F}(\mathrm{y})_{2}=\mathrm{b}_{\mathrm{w} 88}-\mathrm{b}_{\mathrm{j} 88}-\mathrm{b}_{\mathrm{w} 70}+\mathrm{b}_{\mathrm{j} 70} \\
\mathrm{~F}(\mathrm{y})_{1}=\mathrm{b}_{\mathrm{w} 70}-\mathrm{b}_{\mathrm{j} 70}-\mathrm{b}_{\mathrm{w} 39}+\mathrm{b}_{\mathrm{j} 39} \\
\Phi(\mathrm{y})_{2}=\mathrm{b}_{\mathrm{w} 88}-\mathrm{b}_{\mathrm{w} 70} \\
\Phi(\mathrm{y})_{1}=\mathrm{b}_{\mathrm{w} 70}-\mathrm{b}_{\mathrm{w} 39}
\end{gathered}
$$

It was assumed that:

$$
\text { a) } \quad \mathrm{b}_{\mathrm{wxx}}=\left(\mathrm{T}_{\mathrm{eg}}-\mathrm{T}_{2}\right) /[(\mathrm{mf})(\mathrm{rs})]_{\mathrm{wxx}}
$$

$\mathrm{T}_{\text {eg }}$ - gas temperature behind the turbine; $\mathrm{T}_{2}-$ gas temperature in front of the combustion chamber; $\mathrm{mf}$ - mass flow rate $[\mathrm{g} / \mathrm{s}]$; rs - average engine speed; index $\mathrm{w}$ alternative fuel; index $\mathrm{xx}$ - rotational speed, eg. 88,000 rpm; b) $\quad b_{\mathrm{jxx}}=\left(\mathrm{T}_{\mathrm{eg}}-\mathrm{T}_{2}\right) /[(\mathrm{mf})(\mathrm{rs})]_{\mathrm{jxx}}$

index $\mathrm{j}$ - Jet A1 fuel; other signs as above.

The hypothesis that proposed model can be used to describe the influence of different new components on fuels combustion in turbine engine will be verified when:

- values of experimentally determined $\alpha_{i}$ coefficients will be different for fuels containing different components

- difference between $\alpha_{i}$ values for fuels containing different components will correspond with experimentally determined difference between chosen results of combustion process, e.g. $\mathrm{CO}$ concentration in exhaust gases.

\subsection{The results}

The data obtained as the results of stand tests are shown in Table 1 .

Table 1. The average results of tests on MiniJetRig

\begin{tabular}{|l|c|c|c|c|}
\hline Fuel & $\mathrm{rs}$ & $\mathrm{T}_{2}$ & $\mathrm{~T}_{\mathrm{eg}}$ & $\mathrm{mf}$ \\
\hline \multirow{3}{*}{ Jet A1 } & 39.000 & 40.46 & 569.17 & 151.18 \\
\cline { 2 - 5 } & 70.000 & 70.30 & 532.88 & 220.71 \\
\cline { 2 - 5 } & 88.000 & 98.36 & 513.12 & 272.70 \\
\hline \multirow{3}{*}{ Blend No. 1 } & 39.000 & 38.21 & 571.53 & 155.07 \\
\cline { 2 - 5 } & 70.000 & 68.57 & 527.74 & 224.88 \\
\cline { 2 - 5 } & 88.000 & 98.16 & 507.46 & 282.64 \\
\hline Blend No. 2 & 39.000 & 40.59 & 570.18 & 151.65 \\
\cline { 2 - 5 } & 70.000 & 68.89 & 531.19 & 221.31 \\
\cline { 2 - 5 } & 88.000 & 98.25 & 512.23 & 275.15 \\
\hline
\end{tabular}

Basing on the above empirical data the reactivity coefficient $\alpha_{88 / 39}\left(\alpha_{i}\right.$ calculated basing on data for 88,000 , 70,000 , and 39,000 rpm) for each blend was calculated.

Table 2. Results of reactivity coefficient $\alpha_{i}$ calculation for two synthetic components

\begin{tabular}{|l|c|c|}
\hline Blends No & Tested fuel & $\begin{array}{c}\text { The value of reactivity } \\
\text { coefficient } \alpha_{88 / 39}\end{array}$ \\
\hline 1 & $\begin{array}{c}\text { Jet A1 + synthetic } \\
\text { hydrocarbons C15 and C17 }\end{array}$ & 0.241 \\
\hline 2 & $\begin{array}{c}\text { Jet A1 + synthetic } \\
\text { hydrocarbons C8 and C11 }\end{array}$ & 0.740 \\
\hline
\end{tabular}

This model can further be used for determination the relationship between $\alpha_{i}$ and concentration of individual exhaust gas components. The results of $\mathrm{CO}$ measurements are shown in Table 3.

Table 3. The average results of measurements of $\mathrm{CO}$ concentration in exhaust gases

\begin{tabular}{|l|c|c|c|}
\hline Fuel & rs & CO concentr. [ppm] & $\mathrm{CO}_{\mathrm{w}}{ }^{*}-\mathrm{CO}_{\mathrm{j}}{ }^{* *}$ \\
\hline \multirow{4}{*}{ Jet A1 } & 39.000 & 1476.98 & - \\
\cline { 2 - 4 } & 70.000 & 1742.60 & - \\
\cline { 2 - 4 } & 88.000 & 1323.81 & - \\
\hline \multirow{3}{*}{ Blend } & 39.000 & 830.62 & -646.36 \\
\cline { 2 - 4 } & 70.000 & 1044.53 & -698.07 \\
\cline { 2 - 4 } & 88.000 & 513.58 & -810.23 \\
\hline \multirow{2}{*}{ Blend } & 39.000 & 1478.85 & -26.55 \\
\cline { 2 - 4 } & 70.000 & 1716.05 & 401.50 \\
\cline { 2 - 4 } & 88.000 & 1725.31 & \\
\hline
\end{tabular}

${ }^{*} \mathrm{CO}$ concentration in exhaust gases in case the tested blends were used

${ }^{* *} \mathrm{CO}$ concentration in exhaust gases in case the reference Jet fuel was used 
Then, for different engine speeds, the relationship between $\alpha_{88 / 39}$ and $\left(\mathrm{CO}_{\mathrm{w}}-\mathrm{CO}_{\mathrm{j}}\right)$ was established. The results are shown in Fig. 3.

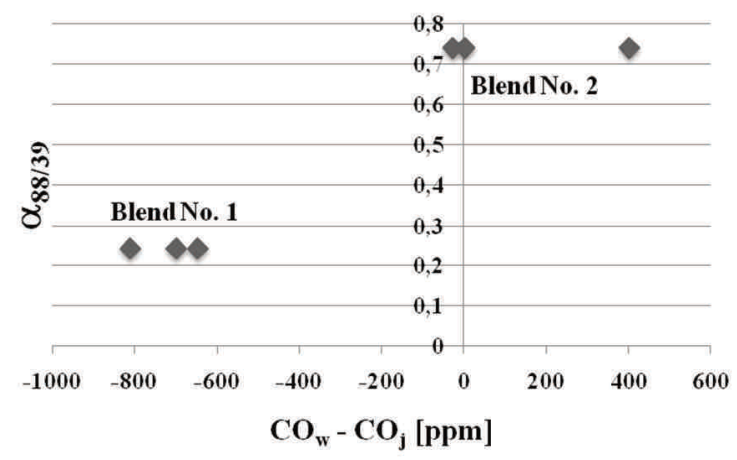

Fig. 3. The relationship between the reactivity coefficient $\alpha_{88 / 39}$ determined for alternative fuels: Blend 1 and 2 in the MiniJetRig engine, and the difference $\left(\mathrm{CO}_{w}-\mathrm{CO}_{\mathrm{j}}\right)$ measured at the engine speed of 39000, 70000 and $88000 \mathrm{rpm}$

\section{Conclusions}

The results shown above lets conclude that:

- values of experimentally determined $\alpha_{88 / 39}$ coefficients are different for fuels containing different components (Blend No. 1 and Blend No. 2)

- difference between $\alpha_{88 / 39}$ values for fuels containing different components correspond with experimentally determined difference between results of combustion process $-\left(\mathrm{CO}_{\mathrm{w}}-\mathrm{CO}_{\mathrm{j}}\right)$.

These results confirm that the proposed model can be used to describe the relation between fuels properties and the results of combustion process.

This is of course a preliminary verification, which leads to further research on this model. Primarily much more fuels should be tested. Than the functional dependence between $\alpha_{88 / 39}$ and $\left(\mathrm{CO}_{\mathrm{w}}-\mathrm{CO}_{\mathrm{j}}\right)$ should be formulated as well as dependences between $\alpha_{88 / 39}$ and concentration of other products of combustion $\left(\mathrm{HC}, \mathrm{PM}, \mathrm{NO}_{\mathrm{x}}\right)$. On the other hand the $\alpha_{88 / 39}$ coefficient should be related to chosen physicochemical properties of the fuels.

\section{Nomenclature}

CI compression ignition

HCCI homogeneous charge compression ignition

$\mathrm{R} \quad$ gas constant $\left(\mathrm{R}=8.3144598(48) \mathrm{J} \cdot \mathrm{mol}^{-1} \cdot \mathrm{K}^{-1}\right)$

\section{Bibliography}

[1] MERKISZ, J. Ekologiczne problemy silników spalinowych. Tom I. Wydawnictwo Politechniki Poznańskiej. Poznań 1998.

[2] GATCHELL, M., ZETTERGREN, H. Knockout driven reactions in complex molecules and their clusters. Journal of Physics B: Atomic, Molecular and Optical Physics. 2016, 49(16), 1-19.

[3] VAnhPVE, G., PETIT, G., MinetTI, R. Experimental study of the kinetic interactions in the low-temperature autoignition of hydrocarbon binary mixtures and a surrogate fuel. Combust. Flame. 2006, 145 (3), 521-532.

[4] TANAKA, S., AYALA, F., KECK, J.C., HEYWOOD, J.B. Two-stage ignition in HCCI combustion and HCCI control by fuels and additives. Combustion and Flame. 2003, 132, 219-239.

[5] MEEKS, E., ANDO, H., CHOUL, C.P. et al. New modeling approaches using detailed kinetics for advanced engines.
The Seventh International Conference on Modeling and Diagnostics for Advanced Engine Systems (COMODIA 2008), 2008, Sapporo, Japan.

[6] PITZ, W.J., CERNANSKY, N.P., DRYER, F.L. et al. Development of an experimental database and chemical kinetic models for surrogate gasoline fuels. SAE Technical Paper. 2007, 2007-01-0175.

[7] SAE International; Aerospace recommended practice ARP 1533C. Procedure for the analysis and evaluation of gaseous emission from aircraft engines.

[8] KAJDAS, C., KULCZYCKI, A., OZIMINA, D. A new concept of the mechanism of tribocatalytic reactions induced by mechanical forces. Tribology International. 2017, 107, 144-151.

[9] GAWRON, B., BIAŁECKI, T. The laboratory test rig with miniature jet engine to research aviation fuels combustion process. Journal of KONBiN. 2015, 4(36), 79-89.

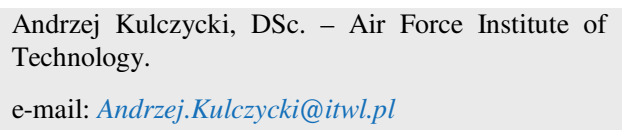

